\title{
TRANSDISCIPLINARY BEHAVIOUR CHANGE: A BURST MODE APPROACH TO HEALTHCARE DESIGN EDUCATION
}

\author{
Anna WOJDECKA ${ }^{1}$, Ashley HALL ${ }^{1}$ and Gaby JUDAH $^{2}$ \\ ${ }^{1}$ Royal College of Art \\ ${ }^{2}$ Imperial College London
}

\begin{abstract}
With the future of health(care) shifting from treatment to prevention, design for behaviour change is an essential part of this movement. Although we have made significant breakthroughs in behavioural science and design for shaping behaviours, there are still some significant gaps and opportunities unexplored. Developing new transdisciplinary approaches to the education of designers for behaviour change becomes of increased importance. New practice-based models are required to facilitate the connection between understanding behavioural theories and applying these to healthcare contexts. This paper illustrates a unique burst mode blended teaching model that fuses teams of mixed healthcare, design, and other diverse backgrounds with the use of digital technologies to pre-empt unwanted behaviour and assist behaviour change. The course module combines asynchronous learning for behaviour change diagnosis with synchronous teaching of behavioural theory, collaborative concept development and rapid prototyping. Digital platforms are used to facilitate remote global teamwork alongside individual physical hands-on prototyping using sensors and electronics.
\end{abstract}

\section{Keywords: Design for behaviour change, blended learning, design innovation, transdisciplinary education}

\section{INTRODUCTION}

Developing new models and tools for designing out unwanted healthcare futures is of paramount importance for healthcare design education and commercial healthcare innovation. New frameworks for design are required, which include an understanding of behavioural science, the impact of the environment on behaviours and how this can be applied in the healthcare contexts through design. Welldesigned products can have a sustained positive effect, e.g., the NHS hospital bed designed by Bruce Archer, which made it easier to tend to patients reducing the strain on nurse's health [1].

Detecting or pre-empting unwanted health behaviour and applying the theory and practice of 'nudging' is still an underexploited approach [2]. Imagine the scale of impact that behavioural therapeutics and preventative solutions designed with the application of new technologies, such as biofeedback and sensors, can have in providing the right healthcare prompts at the right time.

Design for Behaviour Change is one of the core modules in the curriculum of the Healthcare and Design MRes and MSc courses at the Royal College of Art (RCA) and Imperial College London (ICL) [3]. The module is co-delivered in burst mode by both institutions full time over one week and combines lectures, interactive group work and workshops. Alongside an introduction to behaviour change theories and frameworks, the module aims to equip students with hands-on skills to deliver working prototypes. By the end of the module, students are able to design, prototype and present targeted solutions, starting from precisely identifying specific target behaviour, then examining psychological, social, and environmental determinants, and applying behavioural science within the design process. Students develop skills to design interventions for changing behaviour within a specific population or group, targeting healthcare staff, patients, and the general public. Core learning also includes rapid prototyping for concepts articulation, hands-on interactive electronics prototyping, teamwork, and presentation skills.

The Behaviour Change module has been delivered in a face-to-face, highly interactive model in academic years 2018/19 and 2019/20 and as a remote teaching model in 2020/2021. The modules were co-led by two academics representing each collaborating institution, and multiple visiting lecturers 
provided support to students throughout the week. The profiles of teaching staff included design, behavioural science, healthcare, business, electronics, and programming. The cohorts were comprised of students from multiple locations, including the UK, Mexico, Brazil, the US West and East coasts, India, China, Canada, the Netherlands, Greece, Spain and Romania with a variety of previous experience and backgrounds, from social sciences, anthropology, architecture, fashion, fine art, occupational health, emergency medical services, user interaction and product design.

Design for behaviour change can play a crucial role in improving the future of health and healthcare. People's behaviour is still one of the most significant contributors to premature deaths [4]. Design for prevention through shifting behavioural patterns of healthcare staff and patients is one of the largest opportunities to improve health and healthcare outcomes [5].

\section{BEHAVIOURAL FRAMEWORKS AND ELECTRONICS PROTOTYPING}

During the module, students were divided into multidisciplinary teams of two to three students. As a part of module pre-assignment, the RCA students conducted primary and secondary research within the selected challenge area, providing the groundwork for the in-module group work. The challenges included hand-washing in hospitals and public spaces, posture and slouching, recuperation, and recovery, improving body flexibility and gait, sleep regulation and preventing pressure ulcers. For a post-module assignment, the ICL students summarised and analysed the solutions in an essay.

At the start of the module, students received an introduction to behavioural science (including health psychology and behavioural economics approaches), followed by pre-assignment presentations, which summarised conducted research and presented three defined mini-briefs. Teams were formed around selected briefs and focused on applying behaviour change teaching to design practice and a hands-on introduction to electronics work. All groups worked with Arduino Uno boards with sensors kits. Students used Action Actor Context Target Time (AACTT) framework [6] to identify a specific behaviour to change and then understand the determinants of that behaviour, followed by designing ways to overcome barriers and harness facilitators. COM-B model was used to identify whether the target population has the Capability, Opportunity, and Motivation to perform the Behaviour [7, 8]. Behavioural Setting Canvas from Behaviour Centred Design [9] was used to examine the environmental setting and the motives driving behaviour. Students also learned the MINDSPACE framework (Messenger, Norms, Defaults, Priming, Affect, Commitment and Ego) [10] to analyse ways of changing behaviour through changing the environment. Figure 1 shows an example of a behavioural diagnosis assessing the Capability, Opportunity, and Motivation (COM-B) for the behaviour of addressing pressure ulcers in a newly disabled population.

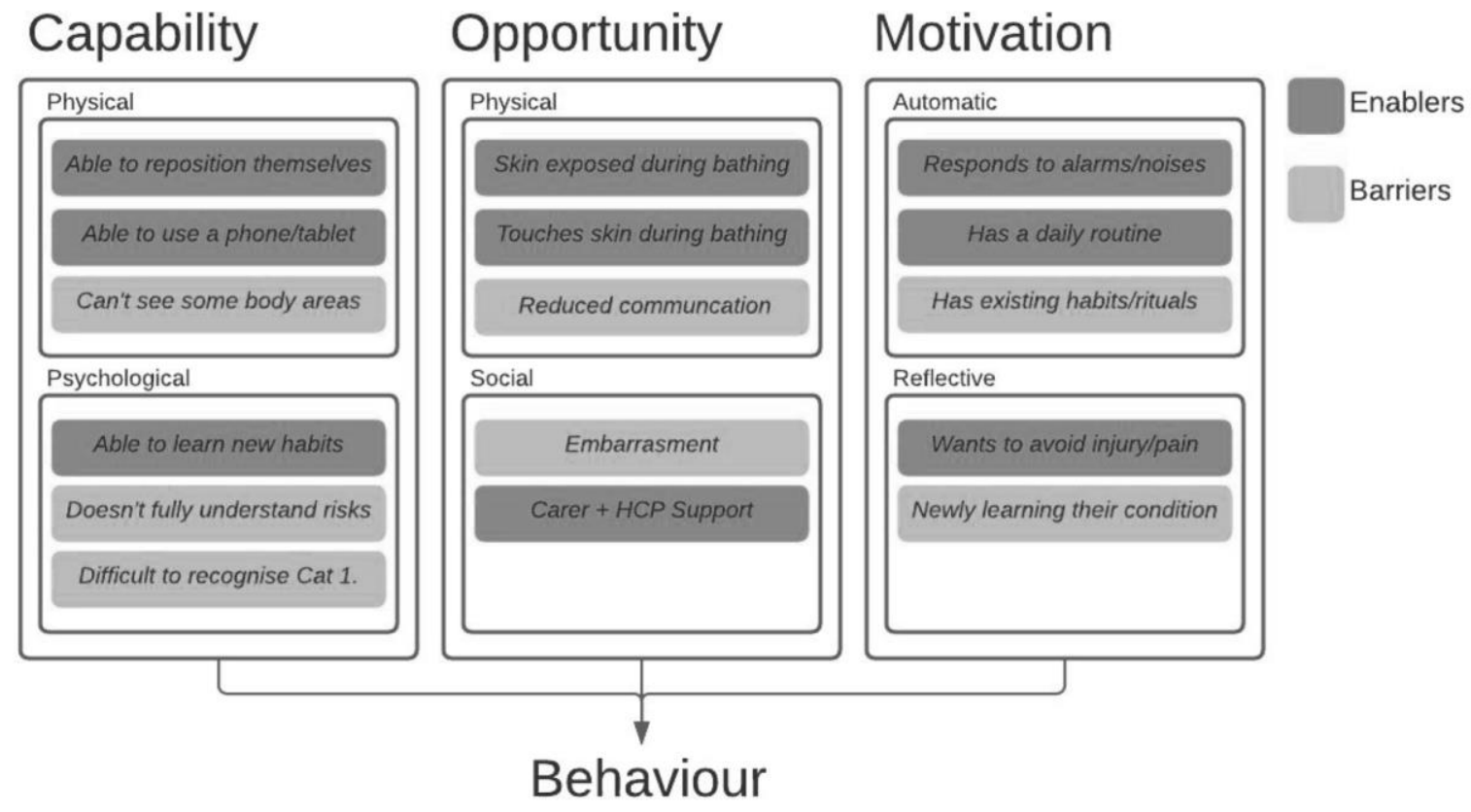

Figure 1. COM-B model analysis within a project addressing pressure ulcers prevention (Cat 1 = category 1 pressure ulcer; $\mathrm{HCP}=$ Healthcare professional) 
Students conducted a brainstorming session with Healthcare and Design Workshop cards [11]. Day four was entirely dedicated to group work and concept development through a 'deep dive' into rapid electronics prototyping workshops. Teams were supported during concept iteration and behavioural context clinics, as well as by the assistance of electronics and programming experts to develop working prototypes (Figure 2.). Students presented the solutions through user-journey scenarios and role-play demonstrations. The practical character of the module prepares students to apply design methods and behavioural theory to promote effective behaviour change in their working lives.

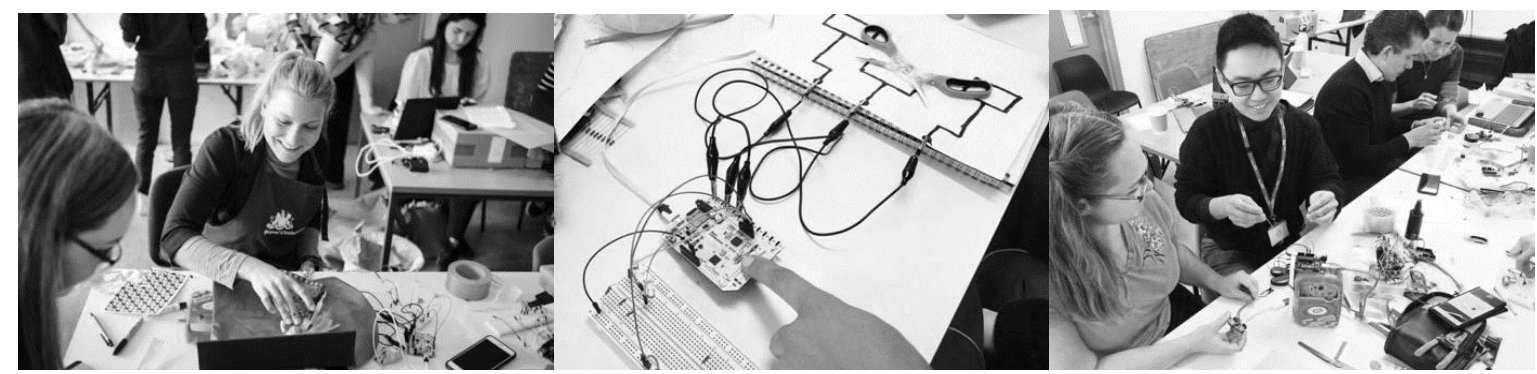

Figure 2. Hands-on electronics prototyping in teams

\subsection{Module Outputs}

Alongside a detailed analysis of the target behaviour, the module outputs covered a wide range of solutions, including products, systems, interactive spaces, or platforms. A selection of prototypes are presented below (Figure 3.). The smart glove dispenser that opens only after correct hand washing completion (Figure 3., left) tackles the challenge of clinical staff wearing gloves without prior hand sanitisation, addressing lack of Automatic Motivation (habit) for sanitising and Physical Opportunity for the unwanted behaviour, through changing Defaults. A car seating system with posture-detection (Figure 3., centre) adjusts to the driver to correct posture and alerts them when they might be falling asleep and need a break (using Salience and Priming). The hand-sanitising bubble dispenser offers a playful way to engage the user in hand disinfection, especially applicable within children's hospital wards and public spaces, such as doors and lift call buttons (increasing Automatic Motivation as the behaviour becomes more enjoyable, thus harnessing Affect). Post-module assignment essays provided an opportunity for critical analysis of the proposed solution and routes to evaluation.

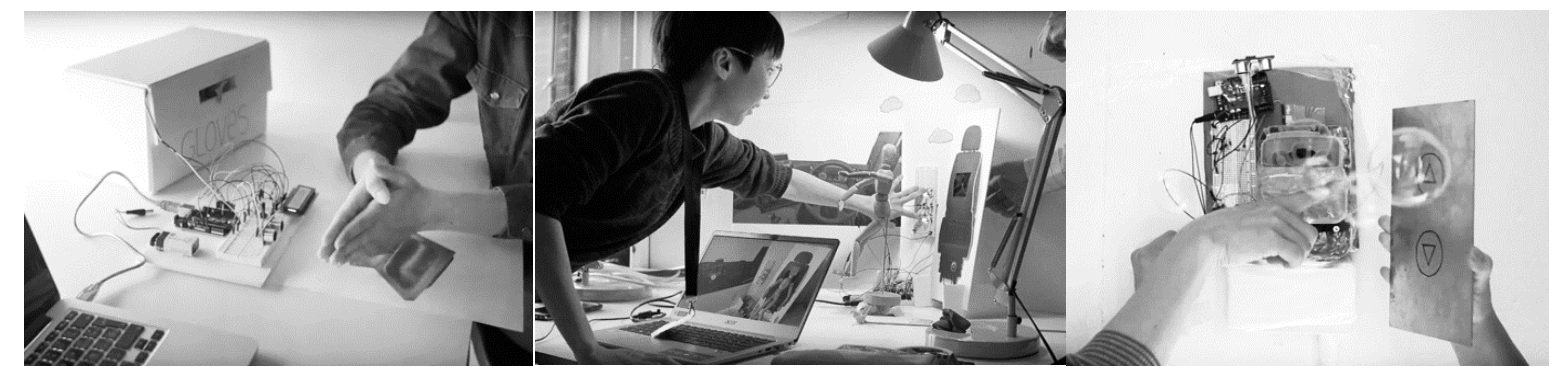

Figure 3. Hand-washing glove dispenser (left), posture-aware correcting seating system (centre) and hand sanitising bubble dispenser (right)

\section{BLENDED STRUCTURE \& ADDRESSED CHALLENGES}

In the academic year 2020/21, the shift to remote teaching and learning due to the global pandemic presented a series of challenges to delivery, requiring rapid module conversion while preserving the module objectives and learning outcomes. The main challenges included:

facilitating teamwork with students and staff from multiple global locations and time zones,

- $\quad$ tailoring module to limit 'Zoom Fatigue', through asynchronous teaching and flipped classroom approach of strategic theory learning facilitated prior to the module, and at the student's convenience,

- $\quad$ sustaining applied module character through physical electronics prototyping,

- team-based opportunity to learn Arduino,

- facilitating collaboration during concept generation development in a virtual space,

- providing an ad-hoc discussion of projects and spontaneous peer-to-peer discussions, 
- $\quad$ building in flexibility to readapt the interactive workshop sessions in real-time when required.

\subsection{Implemented blended approach and structure}

We redesigned the content utilising the flipped-classroom approach to prevent 'Zoom Fatigue' and allow more time for interaction, discussion, and hands-on work during the module. The content was divided into the pre-work assignment and in-module activities. The module pre-work consisted of three types of activities: group work, asynchronous mini-lectures, and electronics skills acquisition.

Twenty students were split into five teams of four participants, maintaining the diversity of backgrounds and compatibility of time zones within each group. The teams were pre-briefed with a challenge area and given the task of mapping the problem using diagramming, selecting specific behaviour and the target population. Using the pre-recorded behaviour change lectures, groups specified the target behaviour (using AACTT) and conducted an initial behavioural diagnosis (understanding the determinants of the behaviour using COM-B). Prior to the module, groups conducted primary and secondary research, including market research of currently available solutions and their success or failure. The electronics introduction was offered as individual pre-work of a series of short 'follow along' tutorials. Each student was provided with a physical Arduino Uno board, Sensors Kit, and instructions for the setup of the Autodesk Tinkercad platform. The module handbook was replaced by a web-based interactive handbook with Zoom links to different activity groups, divided into five colour-coded categories: remote conferencing, hands-on activities, student group work, mentoring timeslots, and electronics support sessions.

As an alternative to physical studio wall space, Miro.com educational whiteboard platform was set up to facilitate group work, visual synthesis, brainstorming and concept articulation. For the hands-on electronics prototyping, each team member used the Arduino physical kit, and groups collaborated on the code development using the Autodesk Tinkercad platform. Each team set up a dedicated project zoom link for the duration of the module, allowing members to virtually 'visit' each other and provide peer-to-peer feedback. The studio-walk-around tutorials were replaced with Global Virtual Studio Patrol, where lecturers joined team rooms for a review using the links provided by teams. In addition, each of the lecturers offered Virtual Open Office Hours to create an opportunity to ask impromptu questions about concept design development, behaviour change aspects, electronics prototyping and coding.

\subsection{Module Outputs}

The outputs were presented during the last day of the module via the Zoom platform and included: a posture tracker for Zoom users, which monitors body position, distance from the screen and provides real-time feedback (Salience) for improvement in sitting position [12] (Figure 3., left), an interactive mat rehabilitation system for post-stroke patients, which uses a gamified approach (Affect, Priming and Norms) to guide users through exercise routine (Figure 3., right), a smart plaster and detection mirror for pressure ulcers detection and prevention (Figure 4.),

- $\quad$ radiographer hand hygiene solution to reduce cross-contamination by utilising a reward system and real-time audio-visual reminders for sanitiser use upon entering a new ward (Automatic Motivation),

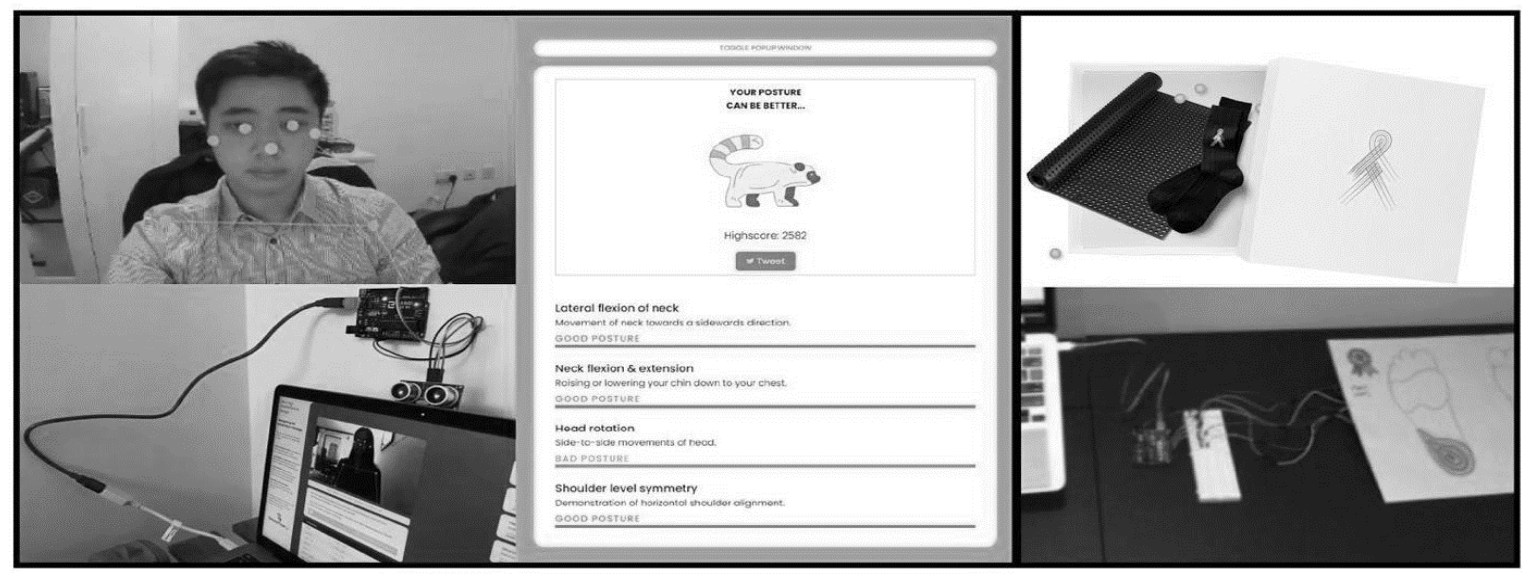

Figure 3. Pouch posture tracker, Walky wearable and smart mat for post-stroke rehabilitation 


\section{REFLECTIONS AND INSIGHTS}

Due to the need for students to work in a highly collaborative way while being physically remote and time zone differences ranging from the US west coast to Asia, we used Zoom and the virtual Miro.com whiteboard. The use of Zoom has become a primary tool for meetings in 2020-2021. However, the Miro.com whiteboard was an extremely effective tool for supporting the creative and collaborative work required for this module. With those measures in place, students were able to work synchronously (for brainstorming and solution ideation) and asynchronously (while working individually on different aspects of the group task). It provided an easy way to collaborate and share ideas with their team members and explain their progress with the module facilitators to receive feedback and advice. Aspects produced on Miro.com were often used within student presentations and reports (Figure 5.).

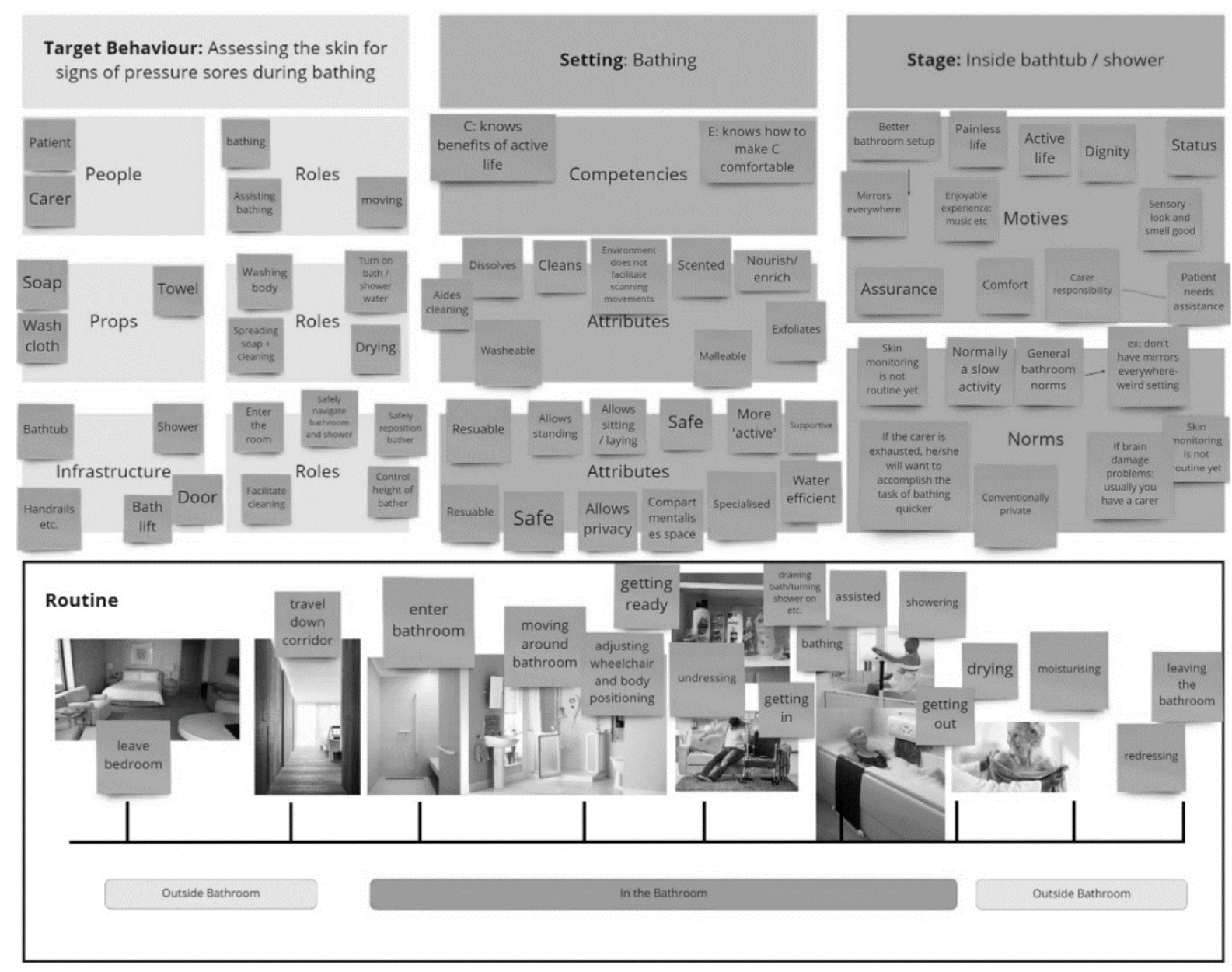

Figure 4. Synthesis - smart plaster and ulcer detection; extract from Miro.com board

The module is delivered in burst mode with an inverted classroom model, including group pre-work before the module, enabling students to "hit the ground running" with their practical group tasks. The pre-work was asynchronous to allow collaboration over different time zones and included mini-lectures (pre-recorded) on behavioural theory and electronics introduction. The RCA students also conducted background work on existing solutions for the target area. Some groups even took the opportunity to conduct interviews or formative research with their target population, based on the concepts taught in the pre-work, which was an added strength for their project. The approach of including pre-work is a realistic parallel with a workplace context, where people may be expected to have reviewed some materials prior to beginning a task. The use of pre-work enabled the best use of the week-long module in terms of collaboration with their team members and learning from one another and getting support from the teaching staff.

The decision to keep the module as a one-week synchronous module rather than convert it to a more extended, asynchronous module (as was done on some other courses) was highly beneficial, as the resulting immersion in the module content and group assignment facilitated intense focus, and 
productive and creative team working. This was demonstrated by the exceptionally high standard of work produced in the final presentations, including the identification of behavioural predictors and application of behavioural theories and methods, exploration of the current solutions and the gap in the existing market, the development of creative solutions to address the identified behavioural predictors, the electronics prototyping and the considered critique of the developed solution.

\section{CONCLUSIONS}

The remote delivery model of the one-week-long burst mode Behaviour Change module provided an excellent opportunity to implement new tools and methods within healthcare design education and further development of the Healthcare and Design MRes and MSc courses. The tools and methods included asynchronous pre-work, synchronous lectures within the module from various experts, tools to facilitate collaborative group work (e.g., Miro.com), and coordinated time for feedback from module staff. The approach used resulted in an effective and enjoyable learning experience for students, demonstrated by positive feedback and excellent work. We were able to facilitate groups to remotely design and make complex physical sensor-based healthcare innovations by breaking the design into subunits that each student made locally in different global geographies, then demonstrated the collective product or service functions online live as a group. We developed processes for asynchronous group work across distant time zones, such as Global Virtual Studio Patrol and Virtual Open Office that mirrored campus-based studio teaching. Miro allowed us much clearer insight into the group process and decision making. Groups found it easier to show the evidence trail for their thinking. Many of those learnings have already been implemented within other modules and commercial healthcare projects and will impact our future curriculum delivery when we return to in-person teaching.

\section{REFERENCES}

[1] Archer B. (1978) Time for a Revolution in Art \& Design Education. RCA Papers, (6).

[2] Vlaev I., King D., Dolan P. and Darzi A. (2016). Theory and practice of 'nudging': Changing health behaviours. Public Administration Review, 76, 550-561.

[3] Hall A., Leff D., Wojdecka A., Kinross J., Thompson P. and Darzi A. 2019. Beyond the healthcare paradigm: Co-creating a new model for collaborative transdisciplinary healthcare design education, E\&PDE 2019, University of Strathclyde, Glasgow.

[4] Schroeder S. A. 2007. We can do better-improving the health of the American people. New England Journal of Medicine, 357(12), pp.1221-1228.

[5] Ryan R. M., Patrick H., Deci E. L., and Williams G. C. (2008). Facilitating health behaviour change and its maintenance: Interventions based on self-determination theory. The European health psychologist, 10(1), pp.2-5.

[6] Presseau J., McCleary N., Lorencatto F., Patey A. M., Grimshaw J. M., and Francis J. J. 2019. Action, actor, context, target, time (AACTT): a framework for specifying behaviour. Implementation Science, 14(1), pp.1-13.

[7] Michie S., Atkins L., and West R. (2014). The behaviour change wheel. A guide to designing interventions. 1st ed. Great Britain: Silverback Publishing, pp.1003-1010.

[8] Michie S., van Stralen M. M. and West R. (2011). The behaviour change wheel: A new method for characterising and designing behaviour change interventions. Implementation Science, 6, 42.

[9] Aunger R. and Curtis V. (2016). Behaviour centred design: towards an applied science of behaviour change. Health psychology review, 10(4), 425-446.

[10] Dolan P., Hallsworth M., Halpern D., King D., and Vlaev I. (2010). MINDSPACE: Influencing behaviour through public policy. Institute for Government, the Cabinet Office. London: England, UK.

[11] Wojdecka A., Hall A., McDonagh R., Leff D., and Kinross J. (2019) Healthcare Design Workshop Cards: Context, People \& Technology, Royal College of Art \& Imperial College London, September 2019, ISBN: 978-0-9561364-5-9.

[12] https://jchui.github.io/posture-tracker/. 